\title{
The genome of herpes simplex virus type 1 is prone to form short repeat sequences
}

\author{
Xiangyan Zhao ${ }^{1 *}$, Xiaolong $\mathrm{Wu}^{1 *}$, Lv Qin ${ }^{2}$, Zhongyang Tan ${ }^{1 \#}$, Shifang $\mathrm{Li}^{2}$, Qingjian Ouyang ${ }^{1}$, \\ You Tian ${ }^{1}$ \\ ${ }^{1}$ College of Biology, State Key Laboratory for Chemo/Biosensing and Chemometrics, Hunan University, Changsha, China \\ ${ }^{2}$ State Key Laboratory of Biology of Plant Diseases and Insect Pests, Institute of Plant Protection, Chinese Academy of Agricultural \\ Sciences, Beijing, China \\ Email: " zhongyang@hnu.edu.cn
}

Received September 2013

\begin{abstract}
Herein, we report a very high content of simple sequence repeats (SSRs) covering $66.12 \%$ of the herpes simplex virus type 1 (HSV-1) genome when a low threshold is adopted to define SSRs, indicating that repeat sequence is a very important character of the HSV-1 genome. The repeats with two iterations account for $68.33 \%$ of the total repeats. In reality, the genome of HSV-1 is prone to form shorter repeat sequences. For mono-, di- and trinucleotide repeats, the repeat numbers decreased with the increase of repeats iterations, implicating that the formation tendency of SSRs might be from low iterations to high iterations. The high iterations SSRs might have subjected to strong selected pressure and survived to perform different functions. The analysis suggested that the repeats formation may be an essential evolutionary driving force for the HSV-1 genome, and the results might be helpful for studying the genome structure, repeats genesis and genome evolution of HSV-1.
\end{abstract}

Keywords: Simple Sequence Repeat; HSV-1 Genome; Microsatellite; SSR

\section{INTRODUCTION}

Herpes simplex virus type 1 (HSV-1) is a member of the Simplexvirus genus in the Herpesviridae family and it is widely distributed in the human population. HSV-1 is the leading cause of viral induced blindness [1]; it gives rise to a variety of clinical disorders and is a major cause of morbidity and mortality worldwide [2]. The global prevalence of HSV-1 is approximately $90 \%$, including $65 \%$ more or less in the USA [3] and 52\% - 67\% in northern

${ }^{*}$ Xiaolong Wu and Xiangyan Zhao are the Co-First Author.

${ }^{\#}$ Corresponding author.
Europe [4]. Like other creatures, the HSV-1 genome contains amounts of repeats. What's interesting is that the content of GC repeats is far high in the HSV-1 genome, which may be related to some pathogenesis of HSV-1 [5]. To our knowledge, the small scope of mutation in repeated regions can cause many diseases [6]; in addition, expansion or contraction of repeats can change the sequence length [7]. Therefore, researching the repetitive sequences we can learn something not only about the interrelationship between genome structure and pathogenesis, but about genome evolution of the HSV-1 from the perspective of molecular biology.

In recent decades, increased attention has been paid to genomic repeat sequences. According to differential lengths of repetitive units, the repeat sequences are usually divided into three basic types: microsatellites, minisatellites and satellite DNA [8]. Mono-, di-, tri-, te- tra-, penta- and hexanucleotide repeats are usually classified as microsatellite-also referred to as short tandem repeats (STRs) or simple sequence repeats (SSRs) [9], and it widely studied in the genomes of prokaryotes and eukaryotes. Repeats of longer units are referred to as minisatellites, the extreme long units, called satellite DNA [6]. Long tandem repeats are observed to be hypermutable, but are rare in exons and only occasionally related with diseases in human $[7,10]$. However, STRs are extremely common and scattered in both coding and non-coding regions in eukaryote [11], prokaryote [12] and also viral [13] genomes. Why are the SSRs so common? Do they perform some functions or are they just "junk DNA" sequences that should perhaps be regarded as "selfish DNA"? Exploring these questions is important to understand how genomes originated, organized and expanded.

In previous studies, microsatellites were researched by using the statistics of relative abundance, relative density, composition and location, etc. In this paper, however, we mainly use repeat iterations to study repeats of short length. Different researchers used different thresholds 
and indicators to identify a motif as a simple repeat, and no real agreement has been reached on this issue; such as someone applied a minimum number of base pairs, whereas, others used a minimum number of repeat units [6]. All these thresholds have made more important contributions in the history of exploring the genome repeat sequences. Here, a relatively low threshold is adopted to study the repeat sequences in the HSV-1 genome, and the analysis may provide new insight into roles of repeat sequences in genome origination, organization and evolution.

\section{MATERIALS AND METHODS}

\subsection{Sequences}

The HSV-1 genome sequence was selected for analysis of relationship between repeat sequence and molecular evolution in the level of genome-wide. The genome sequence with FASTA format was downloaded from the NCBI, and its accession number is NC_001806.1. Additionally, ten genes were randomly selected from the HSV-1 genome in order to make a survey whether the repeats distributed evenly among different fragments.

\subsection{Repeats Extraction}

A program called IMEx (imperfect microsatellite extractor) was selected, which can be used to extract perfect microsatellites [14]. All thresholds of repeat iterations were set to 2 for mono-, di-, tri-, tetra-, penta- and hexanucleotide repeats in this paper. We detected perfect microsatellites using the "Advanced mode" of IMEx. Basic information of SSRs in Supplementary Table 1

Table 1. Percentage of repetitive sequences in the entire genome and ten randomly selected genes of HSV-1.

\begin{tabular}{cccccc}
\hline $\begin{array}{c}\text { Gnome } \\
\text { /Gene }\end{array}$ & Start $^{\text {a }}$ & End & $\begin{array}{c}\text { G. L. } \\
\text { (bp) }\end{array}$ & R. L. (bp) & P (\%) $^{\mathbf{d}}$ \\
\hline $\begin{array}{c}\text { Entire } \\
\text { genome }\end{array}$ & 1 & 152,261 & 152,261 & 100,674 & 66.12 \\
UL2 & 9884 & 10,888 & 1005 & 670 & 66.67 \\
UL8 & 18,224 & 20,476 & 2253 & 1476 & 65.51 \\
UL9 & 20,704 & 23,259 & 2556 & 1563 & 61.15 \\
UL17 & 31,386 & 33,497 & 2112 & 1351 & 63.97 \\
UL25 & 48,813 & 50,555 & 1743 & 1102 & 63.22 \\
UL36 & 71,049 & 80,468 & 9420 & 6178 & 65.58 \\
UL44 & 96,311 & 97,846 & 1536 & 969 & 63.09 \\
UL52 & 109,048 & 112,224 & 3177 & 2049 & 64.49 \\
RS1 & 127,232 & 131,128 & 3897 & 2812 & 72.16 \\
US8 & 141,243 & 142,895 & 1653 & 1056 & 63.88 \\
\hline
\end{tabular}

${ }^{\mathrm{a}}$ Location of genes in the HSV-1 genome; ${ }^{\mathrm{b}}$ Length of genome/gene selected for analysis; 'Length of repeats in the corresponding genome/genes; ${ }^{d}$ Percentage, it equals that the repeats length divide by the lengths of the genome or gene. For example, the percentage of gene UL2 $=670 / 1005=$ $66.67 \%$. and Supplementary Table 2 is referenced in detail.

\section{RESULTS AND DISCUSSION}

When detecting a sequence for microsatellite, definition of the minimum number of repeat iterations is an important empirical criterion. In most previous studies, the minimum number of iterations were set to $6,3,3,3,3$ and 3 for mono-, di-, tri, tetra-, penta- and hexanucleotide repeats respectively $[15,16]$, or a repeat spanned a minimum of 12 nt $[6,9,17]$ or longer [11]. Theoretically, however, as long as the iteration is more than once, the motif should be called a repeat sequence. What's more important, the formation of repeats with high iterations isn't only one step in evolution. Therefore, many shorter repeats were neglected and a large number of useful information were not excavated in their studies. Here, setting the threshold value of all kinds of repeat iterations to 2 , the results suggested that it significantly increased the amount of simple repeats, and the results also have more significance of evolution.

\subsection{Repeat Content in Panoramic Scope}

In order to give a snapshot of various repeats in the entire genome, a fragment was randomly selected from the HSV-1 genome, in which repeats were painted in different color depending on difference of iterations (Figure 1). The figure showed that SSRs were distributed relatively equally throughout the genome and presented a mosaicshaped. Moreover, the longer SSRs (such as pentanucleotide, hexanucleotide repeats...) are more abundant in short and long terminal repeats (TRL and TRS), compared with unique long (UL) and unique short regions (US) (data not shown). The observations are consistent with many previous studies about microsatellites, where they demonstrated that the SSRs distribution is not random in the genome.

Previously, many reports have revealed that a large number of SSRs are located in transcribed regions of genomes, including expressed sequence tags (ESTs) and protein-coding genes [18]. For example, it has been found that: $10 \%$ of identified SSRs in primate [9], 15\% in rabbit [19] located in the open reading frames (ORFs) or protein-coding genes. In protein-coding regions of all known proteins, $14 \%$ proved to contain repeated sequences, with a three times higher abundance of repeats in eukaryotes as in prokaryotes [20]. However, we found 31,705 repetitive sequences in the HSV-1 genome under the criterion of setting the minimum number of iterations to 2 for mono- hexanucleotide repeats (Table 1, Supplementary Table 1). The 31,705 repeat tracts are $100,674 \mathrm{bp}$ in nucleotide length covering $66.12 \%$ of the full genome (Table 1, Supplementary Table 2). In previous study of the HSV-1 genome, a total of only 1377 
Table 2. The count of repeats with different iterations in different repeat motifs of the HSV-1 genome.

\begin{tabular}{|c|c|c|c|c|c|c|c|c|c|c|c|c|}
\hline \multirow{2}{*}{$\begin{array}{l}\text { Repeat } \\
\text { motif }\end{array}$} & \multicolumn{11}{|c|}{ Iterations } & \multirow{2}{*}{ Total } \\
\hline & 2 & 3 & 4 & 5 & 6 & 7 & 8 & 9 & 10 & 11 & $\geq 12$ & \\
\hline Mono- & $\begin{array}{l}\mathbf{1 6 , 0 0 8}^{\mathrm{a}} \\
(62.67)^{\mathrm{b}}\end{array}$ & $\begin{array}{c}\mathbf{5 6 4 6} \\
(22.10)\end{array}$ & $\begin{array}{c}2171 \\
(8.50)\end{array}$ & $\begin{array}{l}1057 \\
(4.14)\end{array}$ & $\begin{array}{c}417 \\
(1.63)\end{array}$ & $\begin{array}{c}181 \\
(0.71)\end{array}$ & $\begin{array}{c}28 \\
(0.11)\end{array}$ & $\begin{array}{c}9 \\
(0.04)\end{array}$ & $\begin{array}{c}8 \\
(0.03)\end{array}$ & $\begin{array}{c}10 \\
(0.04)\end{array}$ & $\begin{array}{c}8 \\
(0.03)\end{array}$ & $\begin{array}{c}25,543 \\
{[80.56]^{c}}\end{array}$ \\
\hline Di- & $\begin{array}{c}3256 \\
(90.60)\end{array}$ & $\begin{array}{c}283 \\
(7.87)\end{array}$ & $\begin{array}{c}43 \\
(1.20)\end{array}$ & $\begin{array}{c}8 \\
(0.22)\end{array}$ & $\begin{array}{c}3 \\
(0.08)\end{array}$ & $\begin{array}{c}1 \\
(0.03)\end{array}$ & - & - & - & - & - & $\begin{array}{c}3594 \\
{[11.33]}\end{array}$ \\
\hline Tri- & $\begin{array}{c}1776 \\
(92.60)\end{array}$ & $\begin{array}{c}119 \\
(6.20)\end{array}$ & $\begin{array}{c}16 \\
(0.83)\end{array}$ & $\begin{array}{c}4 \\
(0.21)\end{array}$ & $\begin{array}{c}2 \\
(0.10)\end{array}$ & $\begin{array}{c}1 \\
(0.05)\end{array}$ & - & - & - & - & - & $\begin{array}{c}1918 \\
{[6.05]}\end{array}$ \\
\hline Tetra- & $\begin{array}{c}370 \\
(95.61)\end{array}$ & $\begin{array}{c}17 \\
(4.39)\end{array}$ & - & - & - & - & - & - & - & - & - & $\begin{array}{c}387 \\
{[1.22]}\end{array}$ \\
\hline Penta- & $\begin{array}{c}\mathbf{1 6 1} \\
(97.58)\end{array}$ & $\begin{array}{c}2 \\
(1.21)\end{array}$ & - & - & $\begin{array}{c}2 \\
(1.21)\end{array}$ & - & - & - & - & - & - & $\begin{array}{c}165 \\
{[0.52]}\end{array}$ \\
\hline Hexa- & $\begin{array}{c}\mathbf{9 4} \\
(95.92)\end{array}$ & $\begin{array}{c}3 \\
(3.06)\end{array}$ & - & - & - & - & - & - & - & - & $\begin{array}{c}1 \\
(1.02)\end{array}$ & $\begin{array}{c}98 \\
{[0.31]}\end{array}$ \\
\hline Total & $\begin{array}{l}21,665 \\
(68.33)\end{array}$ & $\begin{array}{c}6070 \\
(19.15)\end{array}$ & $\begin{array}{l}2230 \\
(7.03)\end{array}$ & $\begin{array}{c}1069 \\
(3.37)\end{array}$ & $\begin{array}{c}424 \\
(1.34)\end{array}$ & $\begin{array}{c}183 \\
(0.58)\end{array}$ & $\begin{array}{c}28 \\
(0.09)\end{array}$ & $\begin{array}{c}9 \\
(0.03)\end{array}$ & $\begin{array}{c}8 \\
(0.03)\end{array}$ & $\begin{array}{c}10 \\
(0.03)\end{array}$ & $\begin{array}{c}9 \\
(0.03)\end{array}$ & 31,705 \\
\hline
\end{tabular}

${ }^{\mathrm{a}}$ The number shown in boldface and italics were neglected in previous studies (in previous studies, a sequence was defined as an repeats when the minimum number of iterations was set to 6, 3, 3, 3, 3 and 3 for mono-, di- , tri-, tetra-, penta- and hexanucleotide repeats, respectively). The summation of these numbers accounts for $96.32 \%(30,539 / 31,705)$ of the total repeats; ${ }^{\text {b }}$ The percentage of repeats with different iterations. For example, when iterations $=2$, the mononucleotides percentage $=16,008 / 25,543=62.67 \%$; ${ }^{\mathrm{c}}$ The percentage of six kinds of repeat motif. For example, the percentage of mononucleotide repeats $=$ 25,543/31,705 = 80.56\%; - Absence of repeats.

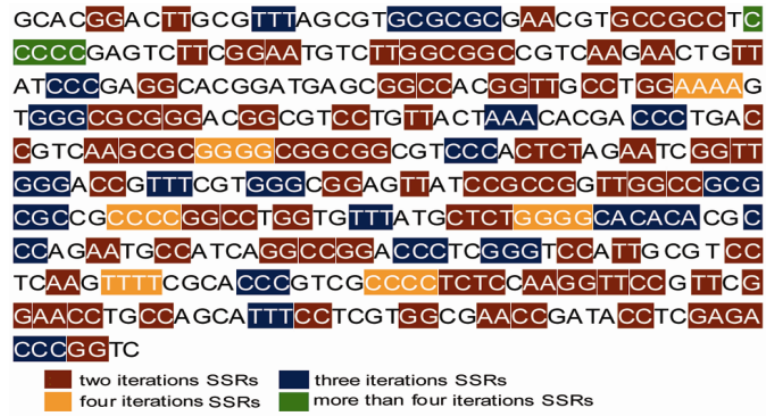

Figure 1. Distribution of repeats in partial genome (Start: 10,441; End: 10,860). All repeat motifs in the sequence that selected randomly from HVS-1 genome were painted in different color depending on different iterations.

repeat tracts were identified [5]. Therefore, considerable parts of the short repeats (approximately 96\%) were neglected in previous studies (Table 2). The repeat content is the highest in gene RS1 and the lowest in UL9 gene; it varies from $60 \%$ to $70 \%$ among the ten genes selected randomly from the HSV-1 genome. These numbers showed that the percentages of repeats were quite high in both genome and genes of HSV-1, and the repeats were widespread in fragments of the genome with little preference.

Obviously, simple repeats are the main components of the genome and they may play important roles in HSV-1. The significantly high content of repeat sequences in the genome indicated that the occurrence of repeats is not random but an essential feature. Therefore, there may be a mechanism existed to make the genome prone to keep repeat sequences in the process of replication. In the long evolutionary history of the HSV-1 genome, the occurrence repeats should be ever more or less than $66.12 \%$, but maybe it holds at this level to face selection pressure and adapt to the environment.

\subsection{The Variation Trend of Repeats}

The two iterations repeats were found to be the most abundant accounting for $68.33 \%$ of the total repeats (Table 2). In detail, the main elements of SSRs with two iterations include about $62.67 \%$ of mononucleotide, and more than $90 \%$ of di-, tri-, tetra-, penta-, and hexanucleotide repeats, respectively. What's more, the repeat numbers decreased with the increase of repeats iterations. In addition, the mononucleotide repeats make up 80.56\% of the total repeats, and repeat numbers also found to decrease with the increase of length of repeat unit. These data clearly showed that the HSV-1 genome tends to form a lot of two iterations repeats, which may be the basis of forming high repetitive sequences, and it also tends to form more simple repeats than complicated repeats in the genome.

From Table 2, we can also summarized that mononucleotide repeats seldom exceeded $10 \mathrm{bp}$ in length, and di-, tri-, and tetranucleotide repeats rarely exceeded 12 bp, penta- and hexanucleotide repeats scarcely ever exceeded 20 bp. Expansions of slippage replication trend to get long of the SSRs, on the contrary, point mutations lead to shorten the long SSRs. One opinion thought that microsatellite repeat length is an equilibrium results from a balance between length and point mutations [21,22]. The existence of upper limits of repeats lengths had 
another explanation that the tendency for repeat length at a locus to rise via mutation is counteracted by selection, and such selection might function through an uncharacterized mechanism on the length of the repeat sequence itself or on gene expression as affected by the SSR sequence at issue [23].

Microsatellite length might be an important factor in affecting mutation rate in HSV-1 genome. The count of SSRs reduces fast with the increase of iterations, and this indicated that it is relatively hard to form repeats with high iterations and long-unit, and these repeats seem to be more instability in HSV-1 genome. However, tetra-, penta- and hexanucleotide repeats were not seriously analyzed in the paper, on account of the low content, compared to mono-, di- and trinucleotide repeats.

\subsection{Short Repeats and Genome Evolution}

The number of repeats with two iterations was 21,665 in the entire genome, accounting for $68.33 \%$ of the total repeats, and they absolutely predominate in the HSV-1 genome (Table 2). In addition, each percentage of two iterations repeats was significantly higher than $90 \%$ except mononucleotides (62.67\%) in the six kinds of repeat motifs, and the summation of repeats with iterations less than 5 accounts for $94.15 \%$ of the total repeats. The two iterations repeats should be subjected to weaker selective pressure in the HSV-1 evolutionary process. High percentage of two times repeats suggested that two times repeats may be the basis of forming repeats with high iterations in the HSV-1 genome. The genome of HSV-1 is prone to form shorter repeat sequences, and these repeats may provide a molecular bias for fast adapting to environmental change in the HSV-1 genome.

\subsection{Long Repeats of Low Frequency}

The exceptions were that there were only two pentanucleotide repeats found with six iterations and one hexanucleotide repeat with more than 12 iterations. The observation of low frequency of longer, complicated repeats possibly result from those repeat sequences are faced with stronger selection pressure than short simple repeats. In the long evolutionary history, the occurrence of long repeat sequences may be lost resulting from harmful or lethal to genome, this may make the genome unable to observe growing longer very quickly. Some short repeats may be neutral to the genomes and been fixed by DNA replication. The remains of long repeat sequences may be benefit to the genomes and have survived from selection pressure to maintain different reported functions [6]. And this repeats prone mechanism possible lead the genome growing longer and longer. This mechanism may explain the observation of the microsatellite polymorphisms deriving mainly from vari- ability in length rather than in the primary sequence [24] and an experimental variant of citrus exocortis viroid (CEVd) expanding its genome with a 96 nucleotide repeat sequences [25]. This is possibly an evidence for the repeat prone mechanism with relation to genome expanding.

\section{CONCLUSION}

By setting low thresholds, the frequency of repeats increased a lot. SSRs were distributed relatively equally throughout the genome and presented a mosaic-shaped; and repeats were quite high in both genome and genes of HSV-1. Simple repeats are the main components of the genome and they may play important roles in HSV-1. HSV-1 genome tends to form a lot of two iterations repeats, which may be the basis of forming high repetitive sequences, and it also tends to form more simple repeats than complicated repeats in the genome. Formation of repeat may be essential for evolution of HSV-1 genome, and the results might be helpful for studying the genome structure, repeats genesis and evolution of HSV-1.

\section{ACKNOWLEDGEMENTS}

This work was supported by the Special project for biodiversity 2012, 2013 of the Chinese ministry of environmental protection and Ministry of Science and Technology torch plan [12C26214304703].

\section{REFERENCES}

[1] Karimi, A. and MacLean, A. (2005) Replication characteristics of herpes simplex virus type 1 (HSV-1) recombinants in 3 types of tissue cultures. Iranian Biomedical Journal, 9, 95-101.

[2] Arduino, P.G. and Porter, S.R. (2006) Oral and perioral herpes simplex virus type 1 (HSV-1) infection: Review of its management. Oral Diseases, 12, 254-270. http://dx.doi.org/10.1111/j.1601-0825.2006.01202.x

[3] Xu, F., Schillinger, J.A., Sternberg, M.R., Johnson, R.E., Lee, F.K., et al. (2002) Seroprevalence and coinfection with herpes simplex virus type 1 and type 2 in the United States, 1988-1994. The Journal of Infectious Diseases, 185, 1019-1024. http://dx.doi.org/10.1086/340041

[4] Pebody, R.G., Andrews, N., Brown, D., Gopal, R., Melker, H.D., et al. (2004) The seroepidemiology of herpes simplex virus type 1 and 2 in Europe. Sexually Transmitted Infections, 80, 185-191. http://dx.doi.org/10.1136/sti.2003.005850

[5] Ouyang, Q., Zhao, X., Feng, H., Tian, Y., Li, D., et al. (2012) High GC content of simple sequence repeats in Herpes simplex virus type 1 genome. Gene, 499, 37-40. http://dx.doi.org/10.1016/j.gene.2012.02.049

[6] Ellegren, H. (2004) Microsatellites: Simple sequences with complex evolution. Nature Reviews Genetics, 5, 435-445. http://dx.doi.org/10.1038/nrg1348

[7] Mirkin, S.M. (2007) Expandable DNA repeats and hu- 
man disease. Nature, 447, 932-940.

http://dx.doi.org/10.1038/nature05977

[8] Ramel, C. (1997) Mini- and Microsatellites. Environmental Health Perspectives, 5, 781-789.

[9] Jurka, J. and Pethiyagoda, C. (1995) Simple repetitive DNA sequences from primates: Compilation and analysis. Journal of Molecular Evolution, 40, 120-126. http://dx.doi.org/10.1007/BF00167107

[10] Sutherland, G.R. and Richard, R.L. (1995) Simple tandem DNA repeat and human genetic disease. Proceedings of the National Academy of Sciences, 92, 3636-3641. http://dx.doi.org/10.1073/pnas.92.9.3636

[11] Toth, G., Gaspari, Z. and Jurka, J. (2000) Microsatellites in different eukaryotic genomes: Survey and analysis. Genome Research, 10, 967-981. http://dx.doi.org/10.1101/gr.10.7.967

[12] Mrazek, J., Guo, X.X. and Shah, A. (2007) Simple sequence repeats in prokaryotic genomes. Proceedings of the National Academy of Sciences, 104, 8472-8477. http://dx.doi.org/10.1073/pnas.0702412104

[13] Zhao, X., Tian, Y., Yang, R., Feng, H., Ouyang, Q., et al. (2012) Coevolution between simple sequence repeats (SSRs) and virus genome size. BMC Genomics, 13, 435. http://dx.doi.org/10.1186/1471-2164-13-435

[14] Mudunuri, S.B. and Nagarajaram, H.A. (2007) IMEx: Imperfect microsatellite extractor. Bioinformatics, 23, 1181-1187. http://dx.doi.org/10.1093/bioinformatics/btm097

[15] Rajendrakumar, P., Biswal, A.K., Balachandran, S.M., Srinivasarao, K., Sundaram, R.M., et al. (2007) Simple sequence repeats in organellar genomes of rice: Frequency and distribution in genic and intergenic regions. Bioinformatics, 23, 1-4. http://dx.doi.org/10.1093/bioinformatics/btl547

[16] Zhao, X., Tan, Z., Feng, H., Yang, R., Li, M., et al. (2011) Microsatellites in different Potyvirus genomes: Survey and analysis. Gene, 488, 52-56. http://dx.doi.org/10.1016/j.gene.2011.08.016

[17] Chen, M., Zeng, G., Tan, Z., Jiang, M., Zhang, J., et al. (2011) Compound microsatellites in complete Escheri- chia coli genomes. FEBS Letters, 585, 1072-1076. http://dx.doi.org/10.1016/j.febslet.2011.03.005

[18] Morgante, M., Hanafey, M. and Powell, W. (2002) Microsatellitesare preferentially associated with nonrepetitive DNA in plant genomes. Nature Genetics, 30, 194200. http://dx.doi.org/10.1038/ng822

[19] van Lith, H.A. and van Zutphen, L.F. (1996) Characterization of rabbit DNA microsatellites extracted from the EMBL nucleotide sequence database. Animal Genetics, 27, 387-395. http://dx.doi.org/10.1111/j.1365-2052.1996.tb00505.x

[20] Marcotte, E.M., Pellegrini, M., Yeates, T.O. and Eisenberg, D. (1999) A census of protein repeats. Journal of Molecular Biology, 293, 151-160. http://dx.doi.org/10.1006/jmbi.1999.3136

[21] Bell, G.I. and Jurka, J. (1997) The length distribution of perfect dimer repetitive DNA is consistent with its evolution by an unbiased single-step mutation process. Journal of Molecular Evolution, 44, 414-421. http://dx.doi.org/10.1007/PL00006161

[22] Kruglyak, S., Durrett, R.T., Schug, M.D. and Aquadro, C.F. (1998) Equilibrium distributions of microsatellite repeat length resulting from a balance between slippage events and point mutations. Proceedings of the National Academy of Sciences, 95, 10774-10778. http://dx.doi.org/10.1073/pnas.95.18.10774

[23] Gur-Arie, R., Cohen, C.J., Eitan, Y., Shelef, L., Hallerman, E.M. et al. (2000) Simple sequence repeats in Escherichia coli: Abundance, distribution, composition, and polymorphism. Genome Research, 10, 62-71.

[24] Mrazek, J. (2006) Analysis of distribution indicates diverse functions of simple sequence repeats in mycoplasma genomes. Molecular Biology and Evolution, 23, 13701385. http://dx.doi.org/10.1093/molbev/msk023

[25] Fadda, Z., Daros, J.A., Flores, R. and Duran-Vila, N. (2003) Identification in eggplant of a variant of citrus exocortis viroid (CEVd) with a 96 nucleotide duplication in the right terminal region of the rod-like secondary structure. Virus Research, 97, 145-149. http://dx.doi.org/10.1016/j.virusres.2003.08.002 